\title{
DNA Double-strand Breaks Induced byFractionated Neutron Beam Irradiation for Boron Neutron Capture Therapy
}

\author{
YUKO KINASHI, NATSUYA YOKOMIZO and SENTARO TAKAHASHI \\ Research Reactor Institute, Kyoto University, Kumatori, Japan
}

\begin{abstract}
Aim: To use the 53BP1 foci assay to detect DNA double-strand breaks induced by fractionated neutron beam irradiation of normal cells. Materials and Methods: The Kyoto University Research Reactor heavy-water facility and gamma-ray irradiation system were used as experimental radiation sources. After fixation of Chinese Hamster Ovary cells with $3.6 \%$ formalin, immunofluorescence staining was performed. Number and size of foci were analyzed using ImageJ software. Results: Fractionated neutron irradiation induced 25\% fewer 53BP1 foci than single irradiation at the same dose. By contrast, gamma irradiation induced $30 \%$ fewer 53BPI foci than single irradiation at the same dose. Fractionated neutron irradiation induced larger foci than gamma irradiation, raising the possibility that persistent unrepaired DNA damage was amplified due to the high linear energy transfer component in the neutron beam. Conclusion: Unrepaired cluster DNA damage was more prevalent after fractionated neutron irradiation than after gamma irradiation.
\end{abstract}

Boron neutron capture therapy (BNCT), which has attracted attention as a therapy that can specifically destroy cancer cells, is usually administered in single irradiation doses. However, when cancer has spread widely, as in malignant pleural mesothelioma or liver tumors, fractionated irradiation is necessary $(1,2)$. Fractionation of radiotherapy is thought to protect normal tissues by allowing repair of sublethal damage (3). In previous studies, sublethal damage repair was not detected in mammalian cells following fractionated high linear energy transfer (LET) irradiation such as carbon beam or thermal neutron irradiation $(4,5)$. In this study, we

Correspondence to: Yuko Kinashi, MD, Ph.D., Research Reactor Institute, Kyoto University, Kumatori-cho, Sennan-gun, Osaka 5900494, Japan. Tel: +81 724512437, Fax: +81 724512623, e-mail: kinashi@ rri.kyoto-u.ac.jp

Key Words: Boron neutron capture therapy, neutron, fractionation, DNA-DSBs. investigated the effect of fractionated thermal and epithermal neutron beam irradiation on normal cells using a colony-formation assay to monitor cell survival rate and 53BP1 foci as markers of DNA damage.

\section{Materials and Methods}

Cell culture. Chinese Hamster Ovary (CHO) K-1 cells (wild-type) were purchased from RIKEN BRC CELL BANK (Tsukuba, Japan). Cells were cultured at $37^{\circ} \mathrm{C}$ in a humidified $5 \% \mathrm{CO}_{2}$ atmosphere in minimum essential media (MEM) alpha medium supplemented with $10 \%$ heat-inactivated calf serum. CHO cells at $100 \%$ confluence in MEM were trypsinized, and $0.8 \mathrm{ml}$ aliquots of cell suspension were placed in Teflon tubes.

Radiation sources and fractionated radiation. The Kyoto University Research Reactor (KUR) heavy-water facility and gammairradiation facility were used as experimental irradiation sources. Fractionated and consecutive irradiation were conducted for both neutron beam and gamma rays. The neutron fluence was measurement from the quantity of radio-activation of the gold foil during the neutron irradiation for BNCT. The gamma-ray dose, including secondary gamma rays, was measured with a $\mathrm{Mg}_{2} \mathrm{SiO}_{4}$ (Tb) thermo-luminescence dosimeter. The physical dose rate was $0.8 \mathrm{~Gy} / \mathrm{h}$. There were four components in the neutron beam: thermal neutrons, 22.4\%; epi-thermal neutrons, $2.4 \%$; fast neutrons, $16.7 \%$; and gamma rays, $58.5 \%$. For fractionated neutron beam irradiation, the first dose was administered to CHO cells in a Teflon tube using a 1.25 Gy neutron beam.

Cell survival assay. $\mathrm{CHO}$ cells were cultured for $23 \mathrm{~h}$ after irradiation. Floating cells were rinsed with phosphate-buffered saline (PBS) before the second neutron irradiation. In the case of fractionated gamma irradiation, the first irradiation of $\mathrm{CHO}$ cells in an Eppendorf tube was performed using 2-Gy gamma rays. The same procedure as used after the first neutron beam irradiation was performed after gamma irradiation. After all irradiations, colonyformation assays were performed to determine the proportion of surviving cells.

53BP1 Foci assay. DNA double-strand breaks (DSBs) in CHO cells following neutron irradiation were detected using the 53BP1 (TP53: tumor suppressor gene p53-binding protein1) foci assay. Immunofluorescence staining was performed as described previously (6). Briefly, irradiated cells were incubated for $3 \mathrm{~h}$, and 




Figure 1. Cell survival curves after neutron radiation (A) and gamma irradiation (B) as a function of radiation dose. Survival curves were fitted by linear regression analysis using all data points. Survival fraction is shown following single-dose $(\mathbf{\Delta})$ and fractionated (৩) irradiation.

Table I. DO and D10 values for Chinese hamster ovary cells irradiated with mixed neutron beam and gamma rays.

\begin{tabular}{lccrrr}
\hline & \multicolumn{2}{c}{ Neutron } & & \multicolumn{2}{c}{ Gamma-ray } \\
\cline { 2 - 3 } & Single irradiation & Fractionated irradiation & & Single irradiation & Fractionated irradiation \\
\hline $\mathrm{D}_{0}(\mathrm{~Gy})$ & 1.1 & 2.3 & 8.8 & 12.4 \\
$\mathrm{D}_{10}(\mathrm{~Gy})$ & 2.7 & 5.4 & 15.2 & 18.8 \\
\hline
\end{tabular}

then washed with cold PBS (Invitrogen). After fixation with $3.6 \%$ formalin, cells were washed with PBS and permeabilized with $0.5 \%$ Triton X-100 in PBS on ice for 5 min. Next, cells were washed with PBS and incubated for $2 \mathrm{~h}$ at $37^{\circ} \mathrm{C}$ with $1 \mu \mathrm{l} / \mathrm{ml}$ primary antibody to $53 \mathrm{BP} 1$ in $5 \%$ skim milk in $1 \times$ TBS buffer, $\mathrm{pH} 7.4$. After three washes with PBS, the cells were incubated for $1 \mathrm{~h}$ at $37^{\circ} \mathrm{C}$ with 1 $\mu \mathrm{l} / \mathrm{ml}$ Alexa Fluor 594 conjugated goat anti-rabbit IgG secondary antibody (Invitrogen, Carlsbad, CA, USA) in TBS-DT solution. The cells were fixed and stained with $10 \%$ glycerol (Wako Pure Chemical Industries Ltd, Osaka, Japan) containing 1\% 4',6diamino-2-phenylindole (Dojindo, Kumamoto, Japan). Immunofluorescence images were acquired on a fluorescence microscope (BZ-9000; KEYENCE, Osaka, Japan), and then analyzed using the BZ-9000 optional software and Image J (National Institutes of Health, Bethesda, MD, USA).

\section{Results}

Figure 1 shows the survival rate associated with each irradiation method. $\mathrm{D}_{0}$ and $\mathrm{D}_{10}$ were calculated from each survival curve (Table I). The results show the difference between consecutive and fractionated irradiation with either neutron beam or gamma rays. The survival rate was higher after fractionated neutron irradiation than after consecutive irradiation. For gamma irradiation, the $\mathrm{D}_{0}$ of fractionated irradiation was 3.6 Gy higher than that of consecutive irradiation. Meanwhile, for neutron beam irradiation, the $\mathrm{D}_{0}$ of fractionated irradiation was $1.2 \mathrm{~Gy}$ higher than that of consecutive irradiation. It is possible that $\mathrm{D}_{0}$ increased due to 
A

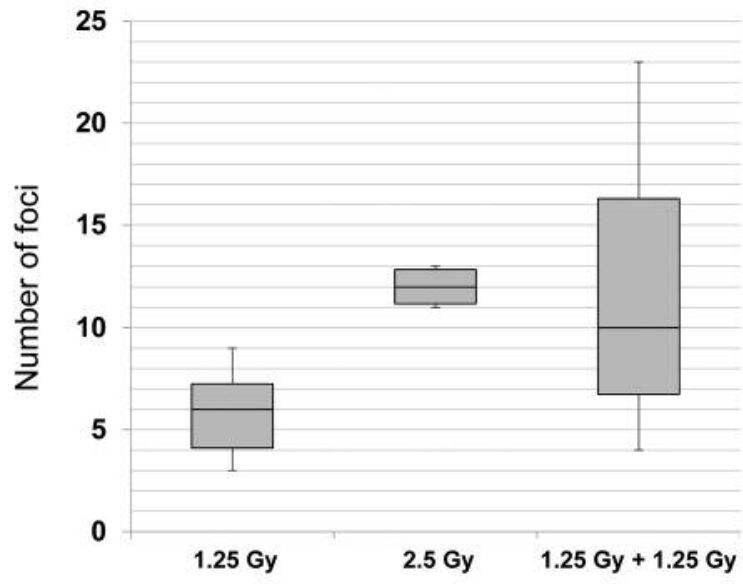

B

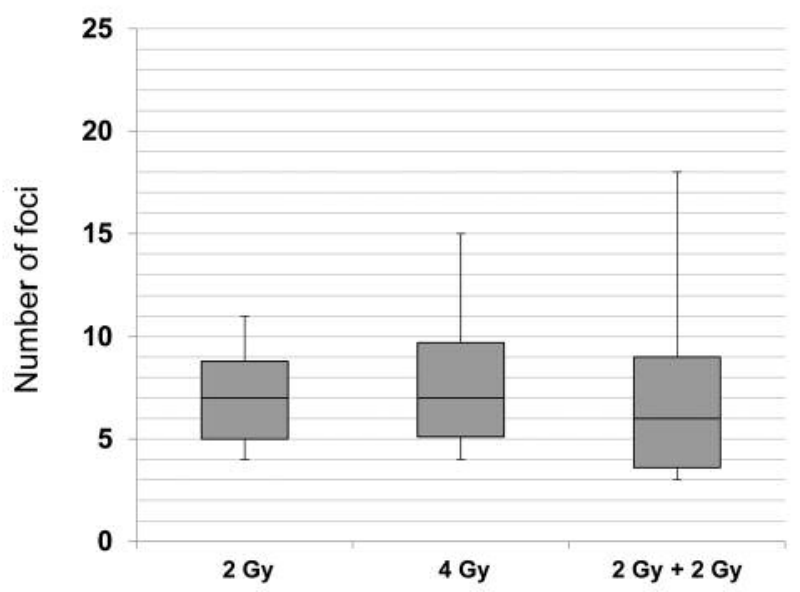

Figure 2. Number of 53BP1 foci per cell $3 \mathrm{~h}$ after fractionated neutron irradiation (A) and gamma irradiation (B).

the selective survival of radiation-resistant cells after the first irradiation. Another possibility is that $\mathrm{D}_{0}$ increased because the radiosensitivity of cells decreased after the first irradiation.

Figure 2 compares the number of 53BP1 foci per cell $3 \mathrm{~h}$ after fractionated neutron beam, and gamma irradiation. In the case of neutron beam irradiation, the variance of foci number was much higher for fractionated irradiation. In the case of gamma irradiation, the median value of fractionated irradiation was somewhat lower than that of consecutive irradiation.

Photographs of 53BP1 foci in the $\mathrm{CHO}$ cells $3 \mathrm{~h}$ after neutron irradiation are shown in Figure 3, and Figure 4 shows the histogram of the size of 53BP1 foci $3 \mathrm{~h}$ after fractionated neutron irradiation or gamma irradiation. Neutron irradiation induced larger 53BP1 foci than did gamma irradiation.

The 53BP1 foci induced by neutron beam irradiation exhibited a different spatial distribution from those induced by gamma rays. Furthermore, the foci induced by fractionated neutron beam irradiation were larger than those induced by a single dose, whereas for gamma irradiation, fractionation did not affect the size of 53BP1 foci.

\section{Discussion}

Fractionation of radiation allows repair of sublethal damage, which is responsible for the increase in survival when a given radiation dose is split into two equal fractions. Utsumi et al. found that sublethal damage repair following thermal neutron therapy was not detected in V79 Chinese hamster cells or B-16 mouse melanoma cells (4). In this study, our results showed that the survival rate was higher after

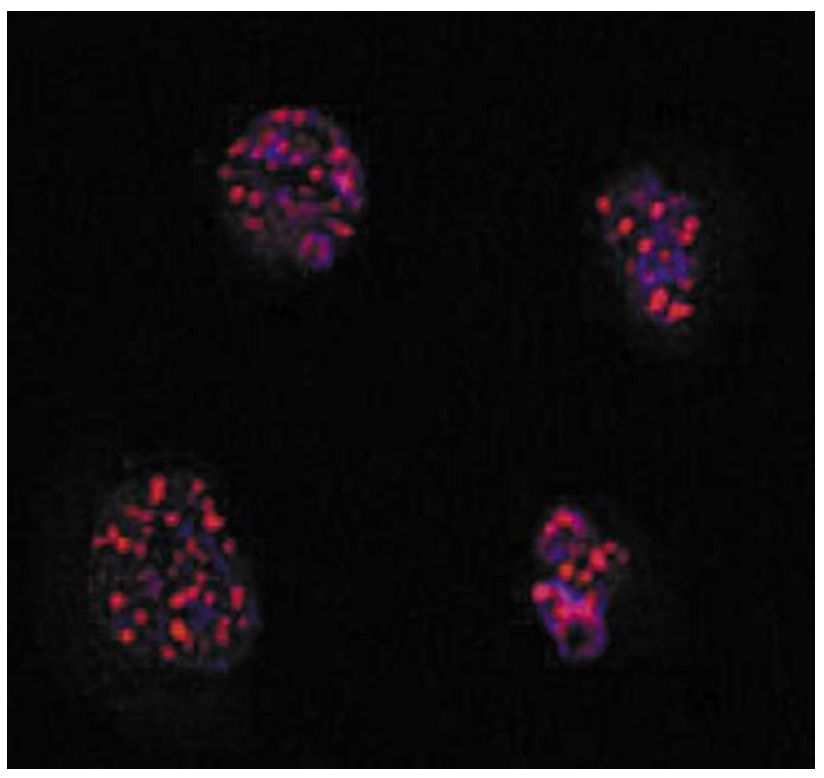

Figure 3. Images of 53BP1 foci in Chinese hamster ovary cells 3 h after neutron irradiation.

fractionated irradiation than after consecutive irradiation, for both gamma and neutron beam irradiation. It is possible that $\mathrm{D}_{0}$ was increased due to the selective survival of radioresistant cells after the first irradiation, or alternatively because the radiosensitivity of the cells decreased after the first irradiation. The KUR neutron beam used for BNCT has a mixed irradiation field. In this experiment, the total physical dose comprised of $22.4 \%$ thermal neutrons, $2.4 \%$ 



Figure 4. Histograms of 53BP1 foci size 3 h after neutron irradiation (A) and gamma irradiation (B). Each bar shows the mean \pm SE size of 53BP1 foci. White bars represent foci after a single dose of irradiation, and black bars represent foci after fractionated irradiation.

epi-thermal neutrons, $16.7 \%$ fast neutrons, and $58.5 \%$ gamma rays. Figure 5 shows the survival fraction when the influence of gamma rays was eliminated. This Figure shows the effect of fractionated irradiation neutron irradiation on survival was not observed in domains less than $0.65 \mathrm{~Gy}$. These results confirm that repair due to fractionated neutron radiation does not occur in normal tissue receiving a low dose of neutrons by BNCT. These results are consistent with those of a previous study (4).

53BP1, an important component of DNA DSBs and repair in mammalian cells, recognizes the unique chromatin environment surrounding DNA DSBs and promotes DSB repair via the non-homologous end-joining pathway (7). In contrast to gamma-H2AX, which accumulates soon after DNA damage, 53BP1 accumulates as a late DNA damageresponse protein and is related to clustered DNA damage (8, 9). Previously, we reported that the 53BP1 foci induced by neutron irradiation were closer to each other and had different spatial distributions from those induced by gamma rays (10). These characteristics of 53BP1 foci are indicative of clustered DNA DSBs $(10,11)$. In this study, we found that fractionated neutron irradiation induced larger foci than did gamma irradiation. It is possible that persistent unrepaired DNA damage is amplified due to the high LET component in the neutron beam.

As far as we are aware of, no previous study has addressed the effect of fractionated neutron irradiation on DNA damage. In the case of gamma rays, fractionated irradiation induced fewer foci than did consecutive irradiation. This result can probably be attributed to DNA repair following gamma irradiation. In the case of neutron irradiation, the variance in foci number was larger following fractionated than consecutive irradiation. It is possible that DNA repair took place due to the gamma rays present in the neutron beam (representing $58.5 \%$ of the total dose, as noted

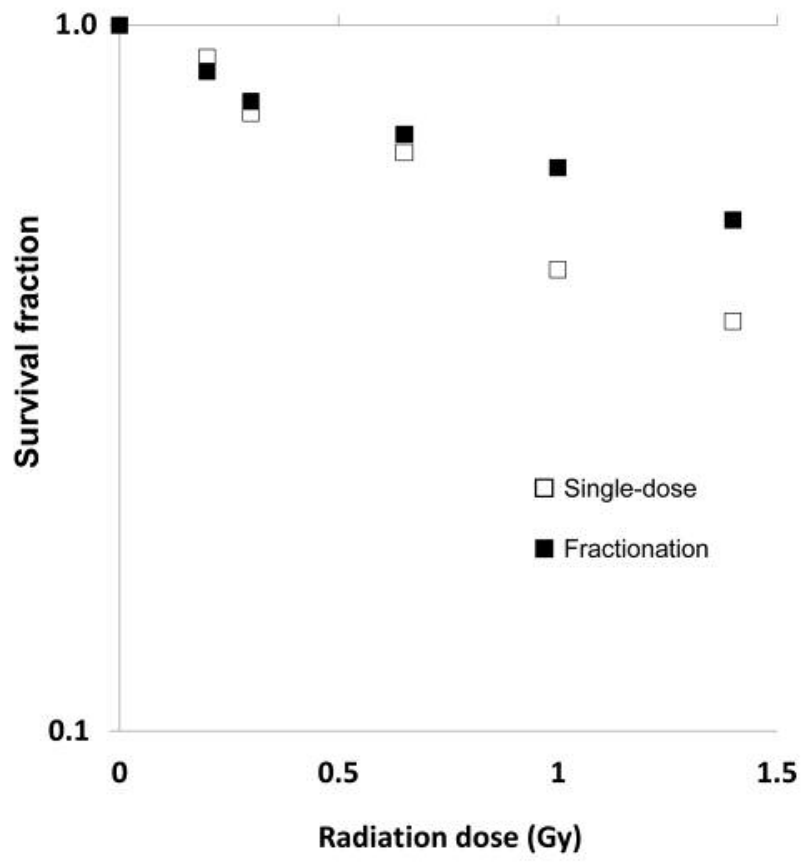

Figure 5. The survival fraction of cells according to the neutron components of the beam for boron neutron capture therapy following fractionated and single-dose irradiation.

above). These results show that fractionated irradiation induces less lethal damage in normal tissue than traditional consecutive irradiation.

Radiation with a high LET component is more effective at inducing biological damage, including cell death and chromosomal aberrations, than low-LET radiation (12). Previous studies have shown that high-LET irradiation induces more complex DNA DSBs than low-LET irradiation (13). 
High-LET irradiation generates highly clustered DNA lesions, increasing the probability of error-prone repair (14). We found that unrepaired clustered DNA damage was more prevalent following fractionated neutron irradiation than following gamma irradiation. After the first neutron irradiation, unrepaired damage persists, and the damage foci grow larger by the second neutron irradiation. These unrepaired DNA lesions may cause radiobiological effects such as genomic instability or mutagenesis.

Secondary carcinogenesis has not been documented during the follow-up of our patients treated with BNCT so far. Since the potential for harm does exist after fractionated irradiation with neutrons for BNCT, long-term follow-up is desirable to monitor for late side-effects, including carcinogenesis, arising in normal tissues.

\section{Acknowledgements}

This study was supported by a Grant-in-Aid for Scientific Research from the Ministry of Education, Culture, Sports, Science and Technology of Japan, No.15K09993 and No.26461884; and by a research grant from Kansai Genshi-ryoku Kondankai, Osaka Japan.

\section{References}

1 Suzuki M, Endo K, Satoh H, Sakurai Y, Kumada H, Kimura H, Masunaga S, Kinashi Y, Nagata K, Maruhashi A and Ono K: A novel concept of treatment of diffuse or multiple pleural tumors by boron neutron capture therapy (BNCT). Radiother Oncol 88 : 192-195, 2008.

2 Suzuki M, Sakurai Y, Hagiwara S, Masunaga S, Kinashi Y, Nagata K, Maruhashi A, Kudo M and Ono K: First attempt of boron neutron capture therapy (BNCT) for hepatocellular carcinoma. Jpn J Clin Oncol 37: 376-381, 2007.

3 Hall Eric J: Time, dose and factionation in radiotherapy. In: Radiobiology for the Radiologist (Third Edition). Philadelphia, J.B. Lippincott Company, pp. 240-259, 1988.

4 Utsumi H, Ichihashi M, Kobayashi T and Elkind MM: Sublethal and potentially lethal damage repair on thermal neutron capture therapy. Pigment Cell Res 2: 337-342, 1989.
5 Wada M, Suzuki M, Liu C, Kaneko Y, Fukuda S, Ando K and Matsufuji N: Modeling the biological response of normal human cells, including repair processes, to fractionated carbon beam irradiation. J Radiat Res 54: 798-807, 2013.

6 Seki K, Kinashi Y and Takahashi S: Influence of p53 status on the effects of boron neutron capture therapy in glioblastoma. Anticancer Res 35: 169-174, 2015.

7 Panier S and Boulton SJ: Double-strand break repair: 53BP1 comes into focus. Nat Rev Mol Cell Biol 15: 7-18, 2014.

8 Schipler A, Mladenova V, Soni A, Nikolov V, Saha J, Mladenov E and Iliakis G: Chromosome thripsis by DNA double strand break clusters causes enhanced cell lethality, chromosomal translocations and 53BP1-recuitment. Nucleic Acids Res doi: 10.1093/nar/gkw487, 2016.

9 Ballarini F, Altieri S, Bortolussi S, Carante M, Giroletti E and Protti N: The role of DNA cluster damage and chromosome aberrations in radiation-induced cell killing: a theoretical approach. Radiat Prot Dosimetry 166: 75-79, 2015.

10 Kinashi Y, Takahashi S, Kashino G, Okayasu R, Masunaga S, Suzuki $\mathrm{M}$ and Ono K: DNA double-strand break induction in Ku80-deficient CHO cells following boron neutron capture reaction. Radiat Oncol 6: 106, 2011.

11 Okumura K, Kinashi Y, Kubota Y, Kitajima E, Okayasu R, Ono $\mathrm{K}$ and Takahashi S: Relative biological effects of neutron mixedbeam irradiation for boron neutron capture therapy on cell survival and DNA double-strand breaks in cultured mammalian cells. J Radiat Res 54: 70-78, 2013.

12 Boei JJWA, Vermeulen S, Mullenders LHF and Natarajan AT: Impact of radiation quality on the spectrum of induced chromosome exchange aberrations. Int J Radiat Biol 77: 847857, 2001.

13 Prise KM: Use of radiation quality as a probe for DNA lesion complexity. Int J Radiat Biol 65: 43-48,1994.

14 Lorat Y, Brunner CU, Schanz S, Jakob B, Taucher-Scholz G and Rübe CE: Nanoscale analysis of clustered DNA damage after high-LET irradiation by quantative electron microscopy. DNA Repair 28: 93-108, 2015.

Received January 17, 2017

Revised March 2, 2017

Accepted March 3, 2017 